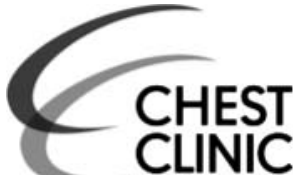

Service de Pneumologie, CHUV, Faculty of Biology and Medicine, University of Lausanne, Lausanne, Switzerland

\section{Correspondence to}

Dr B J Marsland, Service de Pneumologie, CHUV, Faculty of Biology and Medicine, University of Lausanne, Rue du Bugnon 46, BH19.206, Lausanne 1011, Switzerland benjamin.marsland@chuv.ch

Received 16 December 2012 Accepted 20 December 2012 Published Online First 9 January 2013

\title{
Lymphoid follicles in chronic lung diseases
}

\author{
Koshika Yadava, Benjamin J Marsland
}

\section{ABSTRACT}

Lymphoid follicles (LFs) can be induced in the lung on infection or chronic inflammation; however, their relevance and contribution to protective immunity or pathogenesis is poorly understood. Recent advances from clinical studies and animal models have shed some light on the mechanisms that trigger and facilitate the development of LFs. As we grasp a better understanding of their development and their relevance to disease, the potential value in targeting pulmonary LFs with novel therapeutics will become evident.

\section{INTRODUCTION}

The adaptive immune response is initiated in secondary lymphoid organs (SLOs) such as lymph nodes and the spleen. These organs are specialised to enhance interactions between circulating lymphocytes and antigen presenting cells. On encountering antigens specific to their cognate receptors, $\mathrm{T}$ and $\mathrm{B}$ lymphocytes clonally expand within SLOs and are then recruited to the site of inflammation where they exert their effector function. Given the potentially deleterious impact of $\mathrm{T}$ and $\mathrm{B}$ cell function, the formation of SLOs is tightly controlled and occurs during embryogenesis at specific developmental windows and predefined anatomical locations.

However, after birth, de novo formation of ectopic lymphoid follicles (LFs) can occur at mucosal surfaces such as the lung and intestine. Their formation in the lung is associated with infection or inflammation. Accordingly, one could propose that they have emerged to enhance immune responses locally and compliment the functionality of SLOs. However, one could also expect that enhanced local immune responses deriving from LFs could contribute to immune pathology associated with chronic inflammation. LFs within the lung exhibit varying degrees of structural organisation and can be located in association with a bronchiole, pulmonary vessel or in the interstitium. The most organised of these ectopic LFs are referred to as tertiary lymphoid organs and exhibit a structural similarity to SLOs, such as distinct $\mathrm{B}$ and $\mathrm{T}$ cell areas, germinal centres, high endothelial venules and follicular dendritic cell networks.

\section{FACTORS INVOLVED IN THE DEVELOPMENT OF LFS}

There is a certain overlap between the pathways involved in the development of SLOs and inducible LFs. The development of SLOs is dependent on specialised cells called lymphoid tissue inducer (LTi) cells that drive lymphotoxin dependent expression of the homeostatic chemokines CCL19, CCL21 and CXCL13. Although these homeostatic chemokines are also important for the formation of LFs, the necessity for LTi cells in the development of LFs is controversial. Indeed, a recent study by Rangel-Moreno et $a l^{1}$ showed that in neonatal mice, endotoxin induced ectopic pulmonary lymphoid tissue can form in the absence of LTi cells. Instead, T cells producing the cytokine interleukin 17 were sufficient for their formation through induction of lymphotoxin independent expression of CXCL13. Given the association of Th17 cells with chronic inflammation and autoimmunity, the formation of LFs in these conditions could in principle also be a component of interleukin 17 mediated pathology (figure 1).

\section{RELEVANCE OF LFS TO DISEASE}

The formation of pulmonary lymphoid aggregates can be triggered by a variety of stimuli, such as microbial infections and inflammation. Consequently, pulmonary LFs are rarely found in healthy adults whereas they are enriched in chronic disease such as chronic obstructive pulmonary disease (COPD). The landmark paper by Hogg $e t l^{2}$ provided a compelling association between the progression of COPD and the frequency of airways containing LFs, although their role in the disease itself remains elusive. One can speculate that these LFs develop to limit or counteract infection of the airways, which can follow breach of the epithelial barrier in diseased lungs. On the other hand, the breakdown products of the lung tissue that accumulate in the lungs could also be presented within the LFs, giving rise to $B$ and $T$ cell responses directed against self-antigens and thus perpetuate the ongoing disease.

There are conflicting reports regarding the presence of autoantibodies in COPD patients. One of the major caveats in such studies is the definition of an appropriate self-antigen as this may vary, even between subjects exhibiting similar disease severity in terms of clinical symptoms. Moreover, many of these studies measure levels of circulating autoantibodies in the serum of patients. As COPD is seldom linked to systemic autoimmunity, the production of autoantibodies might be localised within the LFs and thus evaluation of their levels within the airways would be more relevant. Van der Strate et $a l^{3}$ have demonstrated that $\mathrm{B}$ cells within pulmonary LFs of COPD patients exhibit an antigen specific oligoclonal activation. Building on the potential for B cell mediated pathology in COPD, Feghali-Bostwick et $a l^{4}$ showed the presence of immune complexes and complement within diseased lungs. In addition, they found increased levels of autoantibodies directed against the 


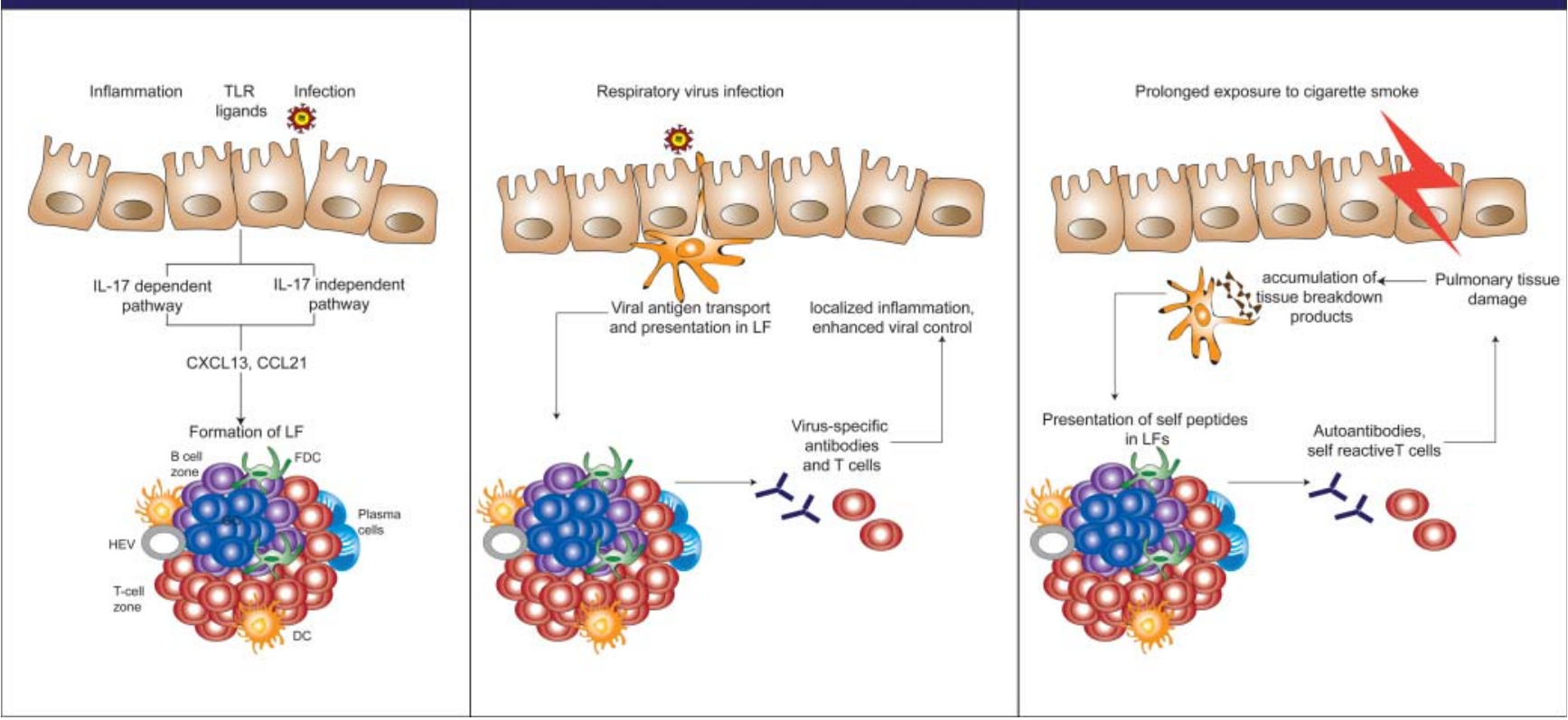

Figure 1 Possible functions and triggers for pulmonary lymphoid follicles (LFs). (A) LF formation is linked with inflammation that is driven by irritants or infections. The chemokines CXCL13 and CCL21 are key participants in the formation and organisation of the follicles. (B) One role of LFs could be to enhance immune responses against pathogens by providing a local site for expansion and differentiation of adaptive immune cells. (C) An alternative, but not mutually exclusive, hypothesis is that LFs form during chronic inflammation and provide a site and reservoir for autoreactive cells that act to enhance disease. DC, ; FDC, ; HEV, ; IL-17, interleukin 17; TLR

pulmonary epithelium that also displayed pathogenic potential, as measured by their ability to mediate antibody dependent cell mediated cytotoxicity. Although these studies allude to a role for LFs in driving immunopathology in COPD, conclusive mechanistic evidence is still lacking.

In addition to chronic inflammation, infections are also known to trigger the formation of LFs. In these situations, LFs could serve as a site for induction of specific immune responses. In fact, such localised responses in addition to being protective might also be less pathogenic in comparison with systemic immune responses. Wiley et $a l^{5}$ studied the role of LFs in the context of protective immunity. They found that induction of LFs by instillation of protein nano cage particles enhances immune responses to primary viral infections while curtailing the extent of bystander damage to the lungs. Of note, this effect mediated by the LFs was not specific and resulted in protection against different viral infections.

\section{CONCLUSION}

It is important to bear in mind that LFs could play a role in protective immunity as well as in immunopathologies. In chronic lung diseases such as COPD, LFs might develop as an intrinsic means of protecting a damaged tissue by enhancing local immune responses; in such cases, therapeutics targeting them could further predispose individuals to infection induced exacerbations. Alternatively, LFs could be key to the pathology and chronicity of COPD, and targeting them could reduce the progression and severity of the disease. Clearly, it is important that we discover the functional role of pulmonary LFs such that rational approaches can be developed to ameliorate the inflammatory environment characteristic of chronic lung diseases.

Contributors This manuscript was written by KY and BJM.

Funding BJM is a Cloetta Medical Research Fellow. This work has been supported by the Swiss National Science Foundation (grant No. 310030.130029).

\section{Competing interests None.}

Provenance and peer review Commissioned; not externally peer reviewed.

\section{REFERENCES}

1 Rangel-Moreno J, Carragher DM, de la Luz Garcia-Hernandez M, et al. The development of inducible bronchus-associated lymphoid tissue depends on IL-17. Nat Immunol 2011;12:639-46.

2 Hogg JC, Chu F, Utokaparch S, et al. The nature of small-airway obstruction in chronic obstructive pulmonary disease. N Engl J Med 2004;350:2645-53.

3 van der Strate BW, Postma DS, Brandsma CA, et al. Cigarette smoke-induced emphysema: a role for the B cell? Am J Respir Crit Care Med 2006;173:751-8.

4 Feghali-Bostwick CA, Gadgil AS, Otterbein LE, et al. Autoantibodies in patients with chronic obstructive pulmonary disease. Am J Respir Crit Care Med 2008; 177:156-63.

5 Wiley JA, Richert LE, Swain SD, et al. Inducible bronchus-associated lymphoid tissue elicited by a protein cage nanoparticle enhances protection in mice against diverse respiratory viruses. PLoS One 2009;4:e7142. 